\title{
Ectopic expression of the apple nucleus- encoded thylakoid protein MdY3IP1 triggers early-flowering and enhanced salt- tolerance in Arabidopsis thaliana
}

\author{
Jian-Qiang Yu ${ }^{1,2}$, Jia-Hui Wang ${ }^{1,2}$, Cui-Hui Sun ${ }^{1,2}$, Quan-Yan Zhang ${ }^{1,2}$, Da-Gang Hu $u^{1,2^{*}+}$ (D) and Yu-Jin Hao $\mathrm{H}^{1,2^{*}+}$
}

\begin{abstract}
Background: The roles in photosystem I (PSI) assembly of the nucleus-encoded thylakoid protein Y3IP1 who interacts with the plastid-encoded Ycf3 protein that has been well-characterized in plants. However, its function and potential mechanisms in other aspects remain poorly understood.

Results: We identified the apple MdY3IP1 gene, which encodes a protein highly homologous to the Arabidopsis Y3IP1 (AtY3IP1). Ectopic expression of MdY3IP1 triggered early-flowering and enhanced salt tolerance in Arabidopsis plants. MdY3IP1 controlled floral transition by accelerating sugar metabolism process in plant cells, thereby influencing the expression of flowering-associated genes. The increase in salt stress tolerance in MdY3IP1-expressing plants correlated with reduced reactive oxygen species (ROS) accumulation, and an increase in lateral root development by regulating both auxin biosynthesis and transport, as followed by enhancement of salt tolerance in Arabidopsis. Overall, these findings provide new evidences for additional functions of Y3IP1-like proteins and their underlying mechanisms of which Y3IP1 confers early-flowering and salt tolerance phenotypes in plants.

Conclusions: These observations suggest that plant growth and stress resistance can be affected by the regulation of the MdY3IP1 gene. Further molecular and genetic approaches will accelerate our knowledge of MdY3IP1 functions in PSI complex formation and plants stress resistance, and inform strategies for creating transgenic crop varieties with early maturity and high-resistant to adverse environmental conditions.
\end{abstract}

Keywords: MdY3IP1, PSI complex, Floral transition, Salt tolerance, ROS, Sugar metabolism

\section{Background}

Growth and development of higher plants largely depends on the photosynthetic activity derived from cooperating photosystem I (PSI) and photosystem II (PSII) complexes [1-3]. PSI is a fundamental pigment-binding protein complex that functions in photosynthetic energy and electron-transfer processes; it uses energy absorbed from sunlight to drive electron transport from plastocyanin to ferredoxin, in cyanobacteria, algae, and plants [4-7]. In higher plants, PSI is protected from

\footnotetext{
*Correspondence: fap_296566@163.com; haoyujin@sdau.edu.cn ${ }^{\dagger}$ Equal contributors

${ }^{1}$ National Key Laboratory of Crop Biology, College of Horticulture Science and Engineering, Shandong Agricultural University, Tai-An, Shandong 271018, China

Full list of author information is available at the end of the article
}

photodamage by a highly efficient antioxidant network that reduces the accumulation of dangerous reactive oxygen species (ROS), by PROTON GRADIENT REGULATION 5 (PGR5)-dependent processes, and by the photoinhibition of PSII [8-10]. Therefore, PSI is considered a robust photosystem.

PSI has a molecular mass of approximately $600 \mathrm{kDa}$, and is composed of two subcomplexes: the PSI core complex and the light-harversting complex I (LHCI). It has at least twelve core subunits in higher plants, including three peripheral (PsaC, PsaD, and PsaE) and nine membrane-intrinsic subunits (PsaA, PsaB, and PsaF-PsaL) [11-14]. Although PSI structure and composition are well characterized, little is known about complex assembly at the membrane [14-16]. Labeling 
experiments indicate that PSI assembly is fast [12, 13], and this makes it difficult to identify additional PSI assembly intermediates.

Although PSI assembly steps remain elusive, several auxiliary protein factors that mediate its biogenesis and assembly in the thylakoid membrane have been identified [16-18]. Two plastid-encoded proteins, Ycf3 (hypothetical chloroplast reading frame number 3) and Ycf4 (hypothetical chloroplast reading frame number 4), are essential for the assembly of the PSI complex [18-21]. The plastidencoded Ycf3 protein also interacts with the nucleusencoded thylakoid protein Y3IP1 (Ycf3-interacting protein) for PSI assembly in Arabidopsis and tobacco [17]. In addition, PPD1 and PSA2 associate with PYG7 are found to regulate the accumulation of PSI, and are involved in PSI biogenesis as well [22-25]. Interestingly, novel PSI assembly factors are constantly discovered, including thylakoid membrane-bound $\mathrm{FtsH}$ proteases and PSA3 [26, 27]. FtsH proteases is responsible for proper biosynthesis of PSI, while PSA3 encoding a protein on the stromal face of the thylakoid membrane promotes the interaction between PSI and assembly factor PYG7 [26, 27].

The Arabidopsis y3ip1 mutant had growth retardation, delayed development, light-green leaf color, and reduction of PSI accumulation phenotypes [17]. Furthermore, Y3IP1 overexpression increased the tolerance to salinity and oxidative stresses in Arabidopsis, leading the gene to be named CHLOROPLAST PROTEIN-ENHANCING STRESS TOLERANCE (CEST) [28]. Despite these phenotypes, AtY3IP1 function in PS1 assembly and roles in other biological processes remain unknown. In this study, we isolated the apple $M d Y 3 I P 1$, a gene with high homology to the Arabidopsis Y3IP1. Ectopic expression of $M d Y 3 I P 1$ triggered early-flowering and enhanced salt tolerance phenotypes in Arabidopsis thaliana. Further analysis found that MdY3IP1 controlled floral transition by accelerating sugar metabolism process in plant cells, and, consequently, altering the expression of floweringassociated genes. MdY3IP1 expression reduced reactive oxygen species (ROS) production and promoted lateral root development by regulating both auxin biosynthesis and transport in salt stressed plants.

\section{Results}

\section{Ectopic expression of the apple chloroplast-localized} protein MdY3IP1 leads to early flowering in Arabidopsis

We identified and cloned the apple MdY3IP1 gene (Accession number: MDP0000930948) (Additional file 1), which encodes a protein highly homologous to the Arabidopsis nucleus-encoded chloroplast-localized AtY3IP1 [17]. To determine the subcellular localization of MdY3IP1, we constructed and transiently expressed pCaMV35S::MdY3IP1-green fluorescent protein (GFP) fusion vectors in protoplasts isolated from apple leaves. A pCaMV35S::GFP plasmid was used as a negative control. The subcellular localization of pCaMV35S::GFP and pCaMV35S::MdY3IP1-GFP fusion proteins was determined using Laser Confocal Microscopy (LCM). The GFP signal of pCaMV35S::MdY3IP1-GFP was enriched at regions that overlapped with chlorophyll auto-fluorescence in the chloroplasts, whereas pCaMV35S::GFP localized throughout the whole cells (Fig. 1a). These results suggest that MdY3IP1 is a chloroplast-localized protein.

To characterize MdY3IP1, we stably transformed Arabidopsis thaliana plants with a construct constitutively expressing MdY3IP1 (35S:::MdY3IP1-myc). An "empty vector $(35 S-m y c)$ " version was used as the negative control. After screening for homozygous seeds, three transgenic lines (MdY3IP1-OE2, MdY3IP1-OE4, and MdY3IP1-OE24) were isolated (Fig. 1b). Analysis of transcript levels by qPCR demonstrated that MdY3IP1 was successfully expressed in all three transgenic Arabidopsis lines (Fig. 1c), while absent in the negative control. Subsequently, we analyzed flowering-related phenotypes of the transgenic plants under long-day and short-day conditions. The number of rosette leaves at flowering was significantly reduced in $M d Y 3 I P 1$ transgenic plants compared to the control (Fig. 1d). Additionally, plants from all three $M d Y 3 I P 1$ transgenic lines bolted earlier than the control (Fig. 1e; Additional file 2).

Taken together, these results indicate that ectopically expressing the chloroplast-localized apple protein MdY3IP1 accelerates floral transition, leading to an early flowering phenotype in Arabidopsis plants.

\section{MdY3IP1 alters the expression of flowering-associated genes by improving photosynthetic derived sugar metabolism}

To further understand how MdY3IP1 regulates flowering in Arabidopsis, we examined the expression of various flowering time-related genes, in control and MdY3IP1-expressing plants. The transcripts of AtSOC1 and $A t F T$, two positive regulators of photoperiodic flowering, were significantly increased in all three $M d Y 3 I P 1$ transgenic Arabidopsis lines (Fig. 2a). Contrarily, the expression of $A t F L C$, a repressor of flowering, was reduced (Fig. 2a).

Various studies have shown that regulation of sugar metabolism contributes to photoperiodic flowering [29, 30]. Furthermore, Y3IP1 plays a key role in photosynthetic carbohydrate synthesis by altering PSI accumulation [17]. Thus, we hypothesized that MdY3IP1 controls flowering by altering starch and soluble sugar content in plants. To verify this hypothesis, we performed iodinestarch staining and UV-spectrophotometry analyses in transgenic Arabidopsis plants. Starch and soluble sugar 

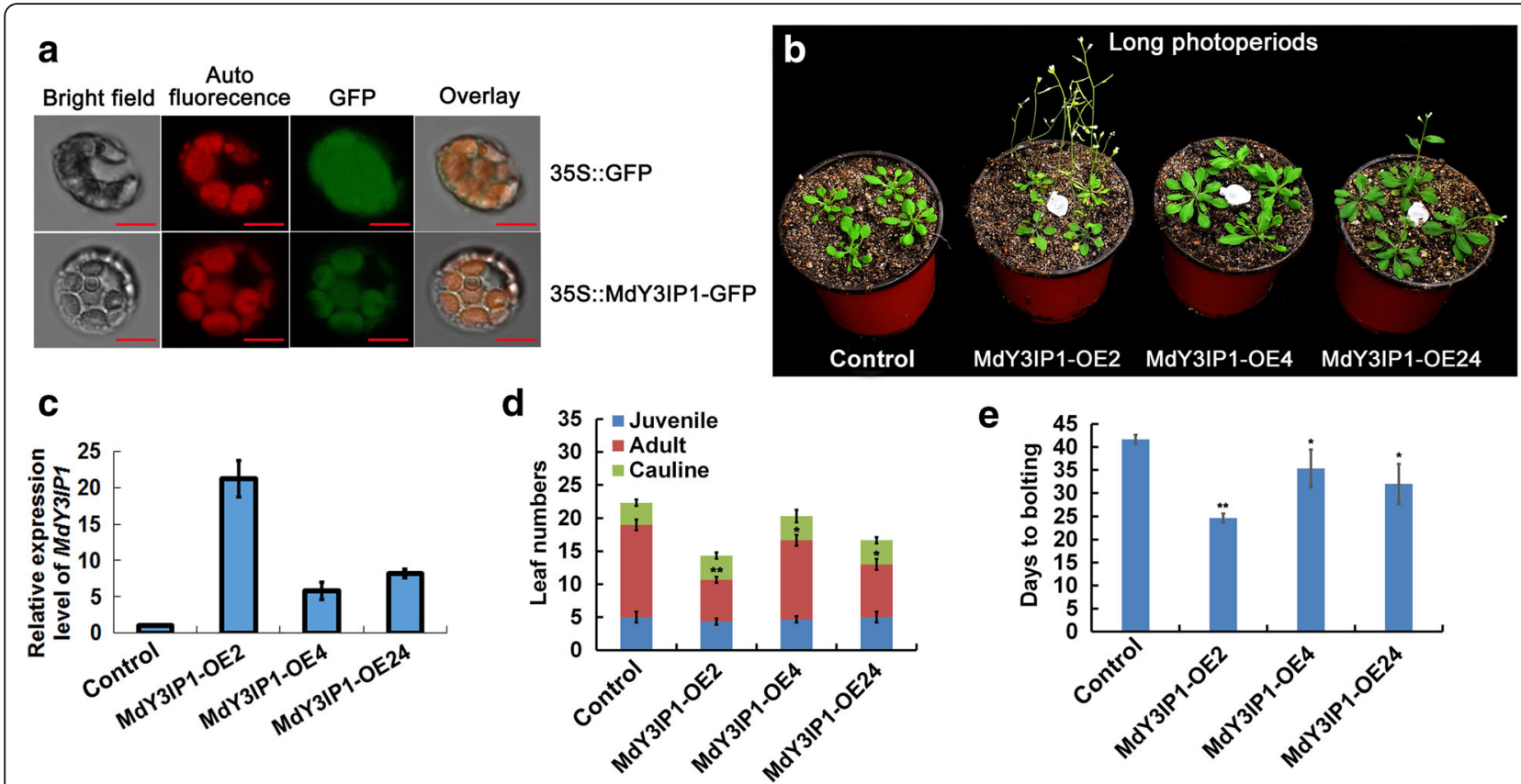

Fig. 1 Early flowering phenotype in the MdY3IP1-expressing transgenic Arabidopsis plants. a Subcellular localization of MdY3IP1. pCaMV35S::MdY3IP1GFP was transiently expressed in protoplasts of apple leaves. pCaMV35S::GFP was used as a negative control. Bars $=100 \mu \mathrm{m}$. b Flowering phenotype of the MdY3IP1 transgenic Arabidopsis. 4-week-old plants grown in soil under long days (LDs) were photographed. Note: Arabidopsis plants transformed with an empty vector serve as the control. c Expression of MdY3IP1 in the control and MdY3IP1 transgenic plants by qPCR assay. Note: Arabidopsis plants transformed with an empty vector serve as the control. $\mathbf{d}$, e Determination of leaf number (d) and of days to bolting (e). Approximate 20 plants grown under LDs were counted and averaged in each assay. Note: In $\mathbf{d}, \mathbf{e}$, data are shown as the mean \pm SE, based on more than nine replicates. Statistical significance was determined using Student's $t$ test. ${ }^{*} P<0.01 ;{ }^{* *} P<0.001$

levels were elevated in all three MdY3IP1 transgenic lines compared to the control (Fig. 2b, c), supporting that $M d Y 3 I P 1$-expression increases sugar contents to influence flowering. We additionally determined the chlorophyll contents, the efficiency of photosynthetic electron transport, and the abundance of PSI core complex subunits in the transgenic plants. As expected, the chlorophyll content was significantly higher in MdY3IP1 transgenic Arabidopsis plants than in the control (Fig. 2d). An increase in the $F_{V} / F_{M}$ (Maximum quantum yield of PSII photochemistry), $\Phi_{I}$ (Effective PSI quantum yield), and $\Phi_{\mathrm{ND}}$ (PSI donor side limitation) was accompanied by a significant decrease of the NPQ (Non-photochemical quenching) in MdY3IP1 transgenic Arabidopsis plants, especially MdY3IP1-OE2 (Fig. 2e, f; Table 1). There was no statistically significant difference in the amount of the PSII reaction center protein D1 between control and MdY3IP1-expressing plants (Fig. 2g). In contrast, the abundance of PSI core complex subunits, including PsaA, PsaD, and PsaF, was much higher in MdY3IP1 transgenic Arabidopsis (Fig. 2g). Collectively, these results suggest that $M d Y 3 I P 1$-expression in Arabidopsis improves photosynthetic carbohydrate synthesis may be mainly by PSI accumulation, consequently altering the expression of flowering-associated genes and accelerating flowering.

\section{MdY3IP1 expression enhances salt tolerance by reducing} ROS accumulation

We analyzed the transcript levels of $M d Y 3 I P 1$ in various apple organs. MdY3IP1 was highly expressed in leaves and flowers, but detected at lower levels in the roots, stems, and fruits (Fig. 3a). Additionally, we investigated changes in MdY3IP1 expression in apple plantlets exposed to various abiotic stresses, including high salinity $(100 \mathrm{mM} \mathrm{NaCl})$, low temperature $\left(4{ }^{\circ} \mathrm{C}\right)$, oxidative stress (2\% Polyethylene Glycol, PEG), and abscisic acid (100 $\mu \mathrm{M}$ ABA). MdY3IP1 transcript levels were induced by all tested abiotic stresses, especially salt stress (Fig. 3b; Additional file 3). These results suggest MdY3IP1 is involved in abiotic stress responses.

To further understand the role of MdY3IP1 in salt tolerance, we challenged MdY3IP1-expressing Arabidopsis plants with $100 \mathrm{mM}$ or $200 \mathrm{mM} \mathrm{NaCl}$. There was no obvious difference in primary root length between the control and MdY3IP1 lines grown on MS medium without $\mathrm{NaCl}$. However, in the plates supplemented with $\mathrm{NaCl}, \mathrm{MdY}$ IIP1 expression lead to a higher salt-tolerance and longer primary roots compared to the control (Fig. 3c, d). Interestingly, the number of lateral roots (LR) was always higher in MdY3IP1 transgenic plants independent of growth conditions (Fig. 3c, e). These results indicated that MdY3IP1 expression enhances salt tolerance in Arabidopsis. 

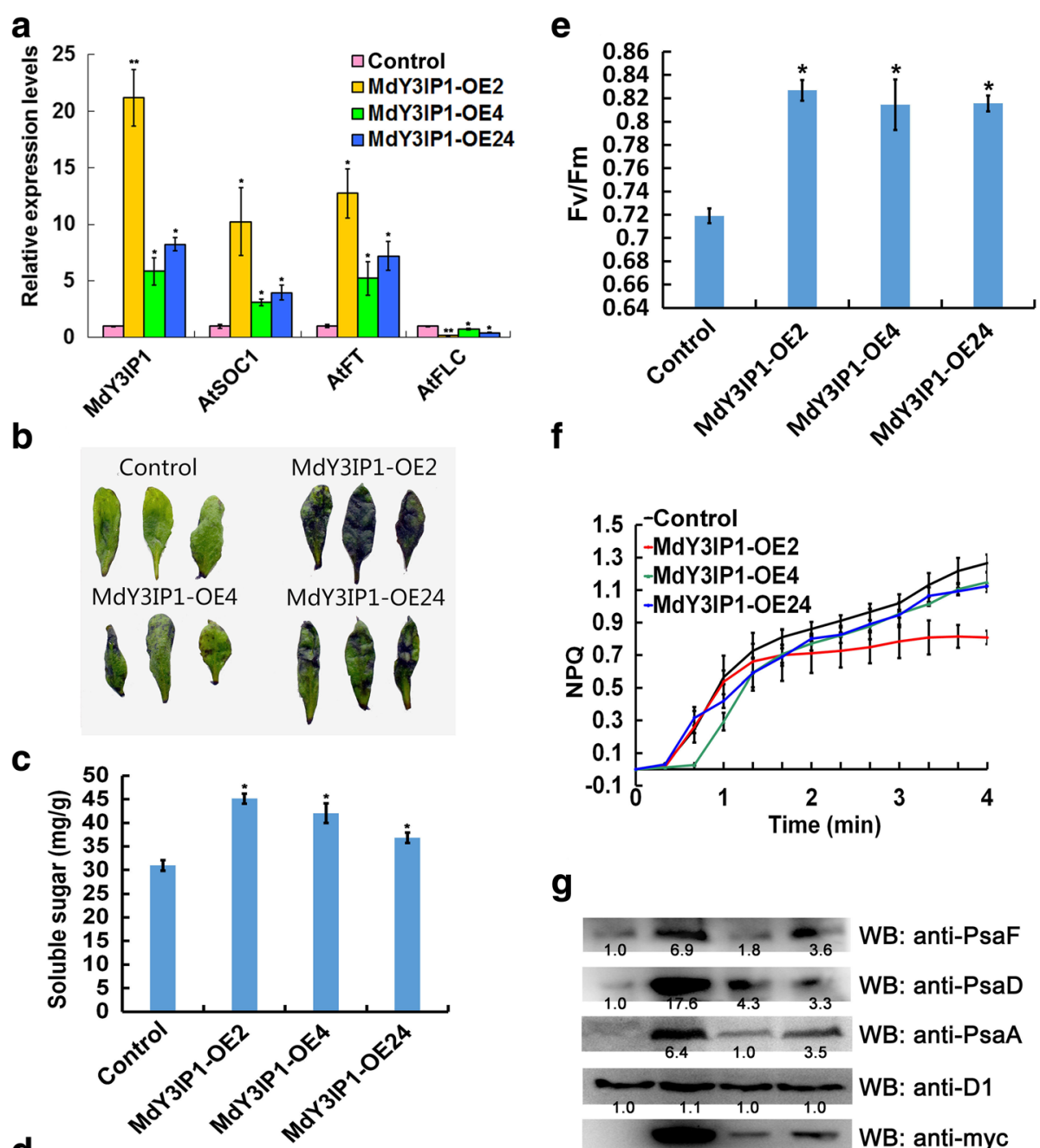

g
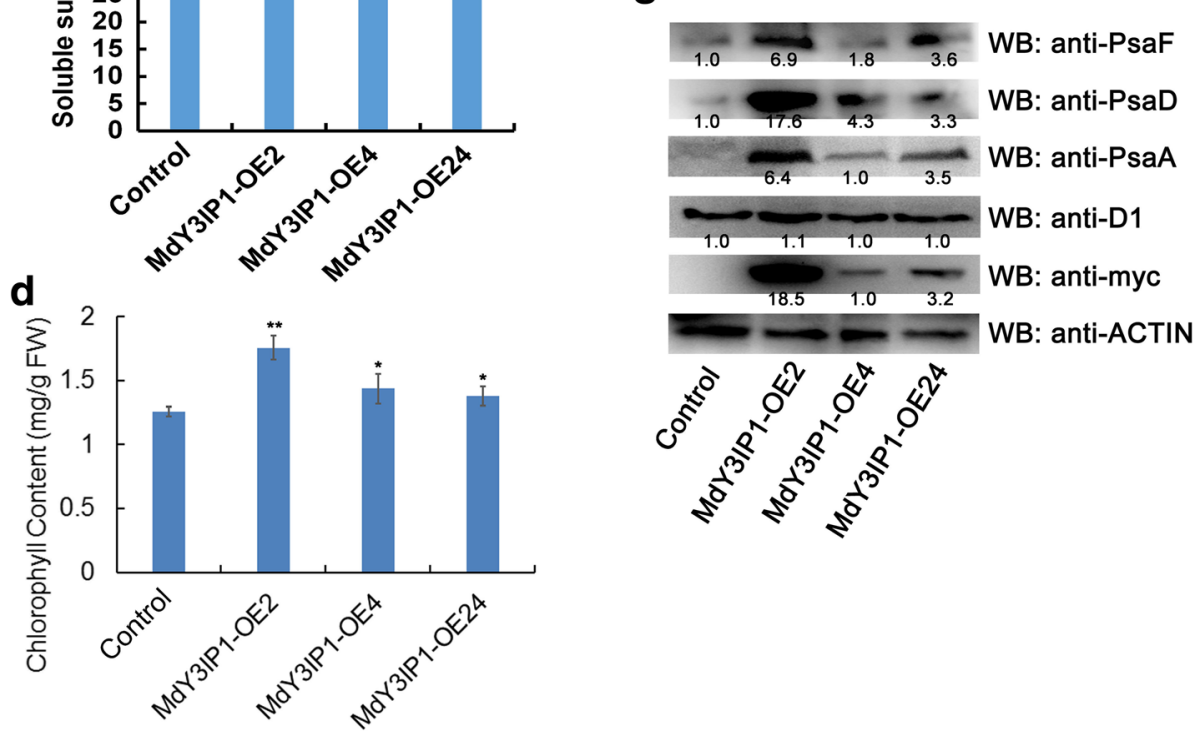

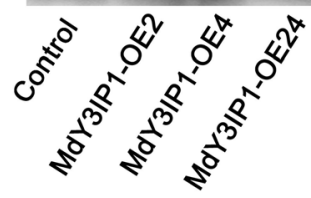

Fig. 2 Photosynthetic sugar metabolism process is accelerated in MdY3IP1 transgenic Arabidopsis. a $\mathrm{PPCR}$ analysis of AtSOC1, AtFT and AtFLC transcript levels in the control and MdY3IP1 transgenic plants. The samplings were occurred around noon during the day time. $\mathbf{b}$ Starch staining of the control and MdY3IP1 transgenic Arabidopsis plants. c Determination of soluble sugars in the control and MdY3IP1 transgenic Arabidopsis plants. $\mathbf{d}$ Chlorophyll content in the leaves of control and MdY3IP1 transgenic Arabidopsis plants. e Maximum quantum yield of PSII (Fv/fm) in the leaves of control and MdY3IP1 transgenic Arabidopsis plants. f Induction and relaxation of NPQ monitored during dark-to-light transition (120 $\mu_{\mathrm{mol}}$ photons $\left.\mathrm{m}^{-2} \mathrm{~s}^{-1}\right)$. Curves represents an average of six independent measurement. $\mathbf{g}$ Immunoblots of PSI core protein subunits including PsaA, PsaD, and PsaF, as well as PSII reaction center protein D1 in the control and MdY3IP1 transgenic Arabidopsis plants. An anti-myc antibody was used to detected protein abundance of MdY3IP1-myc transgenic Arabidopsis. Anti-ACTIN antibody was used as a negative control. Note: In $\mathbf{a}, \mathbf{c}, \mathbf{d}$, e, data are shown as the mean \pm SE, based on more than nine replicates. Statistical significance was determined using Student's $t$ test. ${ }^{*} P<0.01 ;{ }^{* *} P<0.001$ 
Table 1 Functional characteristics of the thylakoid membrane of WT (col) and MdY3IP1 (MdY3IP1-OE2, MdY3IP1-OE4 and MdY3IP1-OE24) transgenic Arabidopsis plants. Values were measured from plants grown under moderate light intensities $\left(120 \mu \mathrm{mol}\right.$ photons $\left.\mathrm{m}^{-2} \mathrm{~s}^{-1}\right)$

\begin{tabular}{lllll}
\hline Photosynthetic parameter & WT (col) & MdY3IP1-OE2 & MdY3IP1-OE4 & MdY3IP1-OE24 \\
\hline Fraction of oxidizable PSI, PM & $0.12 \pm 0.01$ & $0.16 \pm 0.01^{*}$ & $0.12 \pm 0.01$ & $0.13 \pm 0.02$ \\
Effective PSI quantum yield, ФI & $0.38 \pm 0.02$ & $0.45 \pm 0.03^{*}$ & $0.41 \pm 0.01^{*}$ & $0.46 \pm 0.03^{*}$ \\
PSI donor side limitation, ФND & $0.52 \pm 0.02$ & $0.63 \pm 0.03^{*}$ & $0.56 \pm 0.01^{*}$ & $0.56 \pm 0.02$ \\
PSI acceptor side limitation, ФNA & $0.10 \pm 0.01$ & $0.11 \pm 0.02$ & $0.11 \pm 0.02$ & $0.10 \pm 0.01$ \\
Effective PSI quantum yield, ФII & $0.25 \pm 0.03$ & $0.24 \pm 0.05$ & $0.27 \pm 0.03$ & $0.30 \pm 0.03$ \\
Yield of non-regulated non-photochemical energy loss, DNO & $0.33 \pm 0.02$ & $0.39 \pm 0.07$ & $0.34 \pm 0.02$ & $0.33 \pm 0.01$ \\
Non-photochemical energy dissipation, DNPQ & $0.42 \pm 0.01$ & $0.32 \pm 0.04^{*}$ & $0.39 \pm 0.01^{*}$ & $0.37 \pm 0.02^{*}$ \\
Excitation pressure of PSII, 1-qP & $0.40 \pm 0.05$ & $0.41 \pm 0.02$ & $0.42 \pm 0.04$ & $0.42 \pm 0.01$ \\
\hline
\end{tabular}

The values are the means \pm SD, $n=6-9$. Statistically significant differences comparing the MdY3IP1 transgenic Arabidopsis plants to that of the corresponding WT (col) are marked with asterix $(*)$. See text for details. WT, wild-type

It is widely known that high salinity induces oxidative stress [31, 32]. Therefore, we examined ROS accumulation in control and MdY3IP1 transgenic Arabidopsis plants. We compared the production of $\mathrm{H}_{2} \mathrm{O}_{2}$ (DAB staining) and of superoxide (NBT staining) in control and MdY3IP1-expressing Arabidopsis leaves, and found that MdY3IP1 plants accumulated less of ROS species in both salt-treated and untreated populations (Fig. 4a-d). DCFH-DA staining was also used to detect the accumulation of $\mathrm{H}_{2} \mathrm{O}_{2}$. The fluorescent signal corresponding to $\mathrm{H}_{2} \mathrm{O}_{2}$ was also much lower in $M d Y 3 I P 1$-expressing cells, confirming that MdY3IP1 reduced ROS production in Arabidopsis (Fig. 4e, f). These results suggest that MdY3IP1expression enhanced salt tolerance in Arabidopsis by reducing ROS accumulation in cells.

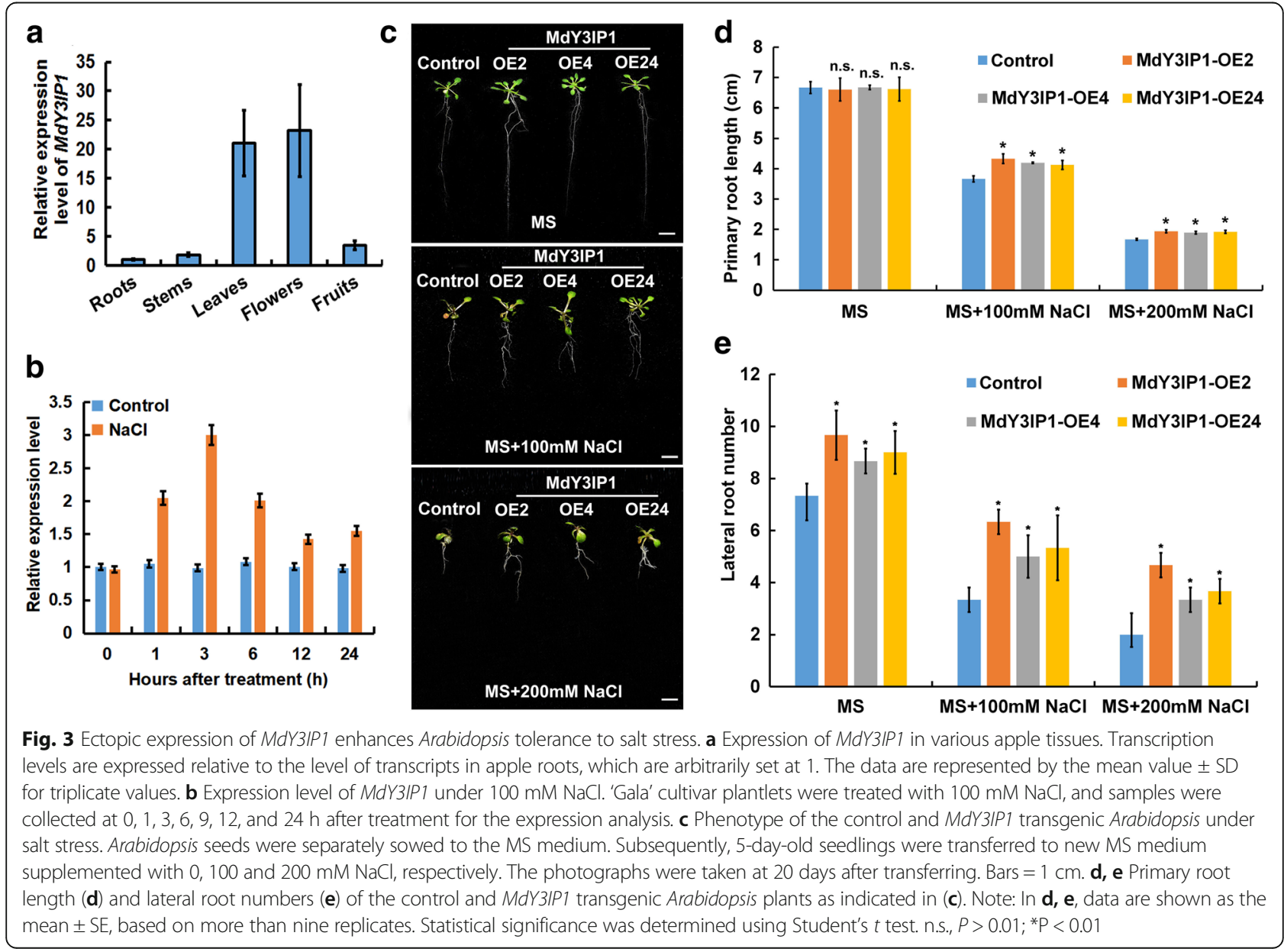


a

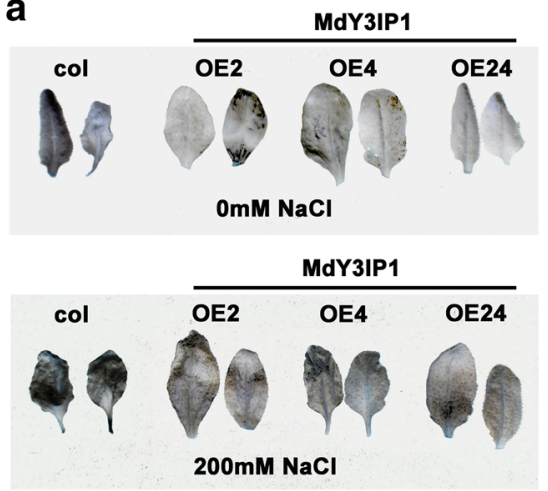

C
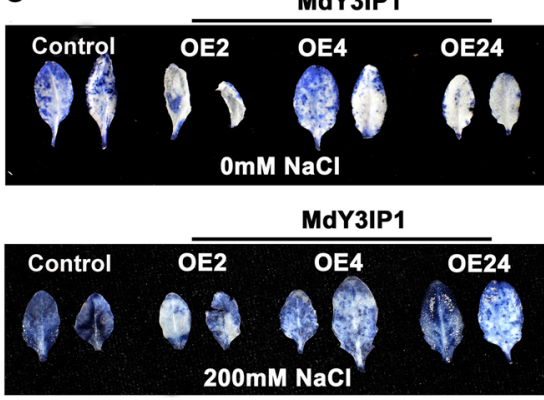

e

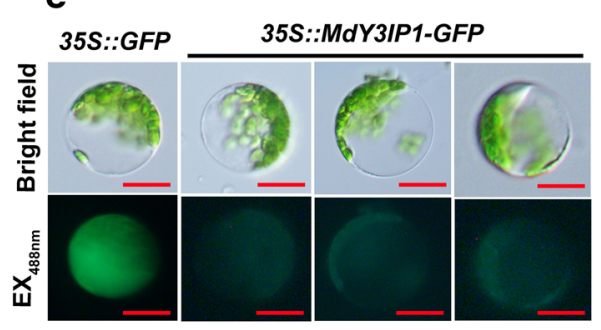

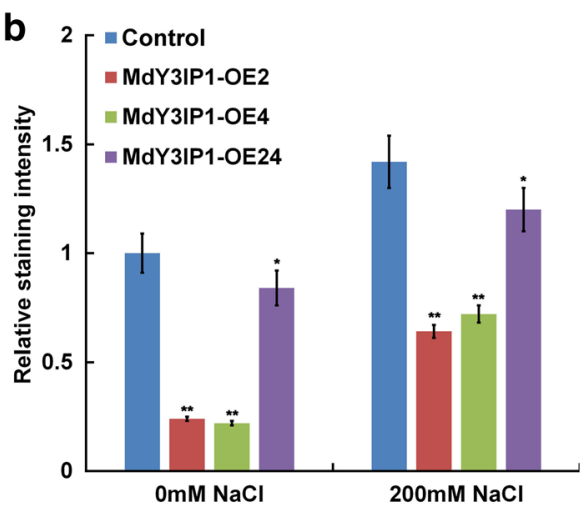

d

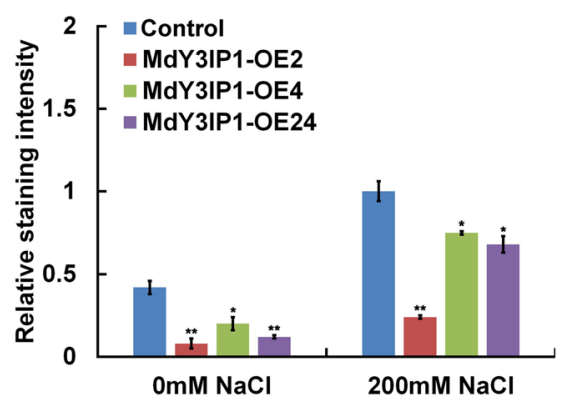

f

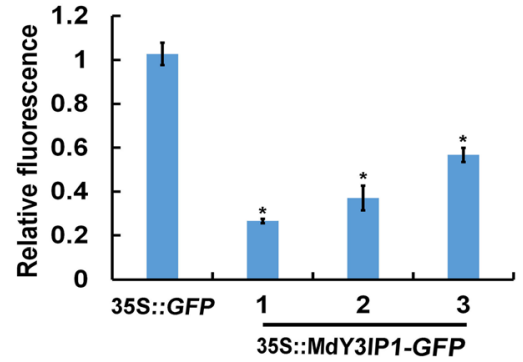

Fig. 4 MdY3IP1 accumulates less ROS than the control in Arabidopsis leaves. a DAB staining for $\mathrm{H}_{2} \mathrm{O}_{2}$ in the leaves of the control and MdY3IP1 transgenic Arabidopsis grown with or without a $200 \mathrm{mM} \mathrm{NaCl}$. b DAB staining intensity as determined with imageJ software. $\mathbf{c}$ NBT staining for superoxide in the leaves of the control and MdY3IP1 transgenic Arabidopsis grown with or without a $200 \mathrm{mM} \mathrm{NaCl}$. d NBT staining intensity as determined with imageJ software. e DCFH-DA staining for $\mathrm{H}_{2} \mathrm{O}_{2}$ in the leaf protoplasts of the control and MdY3IP1 transgenic Arabidopsis treated with or without $200 \mathrm{mM} \mathrm{NaCl}$. Bars $=100 \mathrm{~mm}$. f DCFH-DA staining intensity as determined with imageJ software. Three independent experiments were done with similar results, each with three replicates, and each replicate with 20 to 30 protoplasts. Note: In $\mathbf{b}, \mathbf{d} \mathbf{f}$, data are shown as the mean $\pm \mathrm{SE}$, based on more than nine replicates. Statistical significance was determined using Student's $t$ test. ${ }^{*} P<0.01 ;{ }^{* * P}<0.001$

MdY3IP1 promotes LR development by influencing local auxin biosynthesis and polar transport

Extensive research has shown that multiple hormonal pathways and environmental conditions influence LR initiation and development by regulating auxin homeostasis, biosynthesis, and transport [33]. Hence, we determined the total auxin content in control and MdY3IP1 transgenic Arabidopsis plants. The MdY3IP1-expressing plants accumulated more auxin than the control whether it was on normal MS medium or on MS medium supplemented with $100 \mathrm{mM} \mathrm{NaCl}$ (Fig. 5a). Moreover, the transcript levels of auxin influx carriers (AtAUX1), efflux carriers (AtPIN1, AtPIN2, and AtPIN3), and biosynthetic YUCCA genes (AtYUC1, AtYUC2, and AtYUC6) were increased in MdY3IP1-expressing plants compared to the control (Fig. 5b). This implies that MdY3IP1 promotes LR development by regulating auxin biosynthesis and polar transport.

To further confirm this possibility, we grew seedlings on MS medium supplemented with the auxin transport inhibitor N-1-naphthylphthalamic acid (NPA). Application of $10 \mu \mathrm{M}$ NPA significantly inhibited LR numbers in both control and MdY3IP1 transgenic plants. However, LR numbers in MdY3IP1 transgenic plants slightly increased compared to the control under this condition (Fig. 5c), whereas no significant difference in 

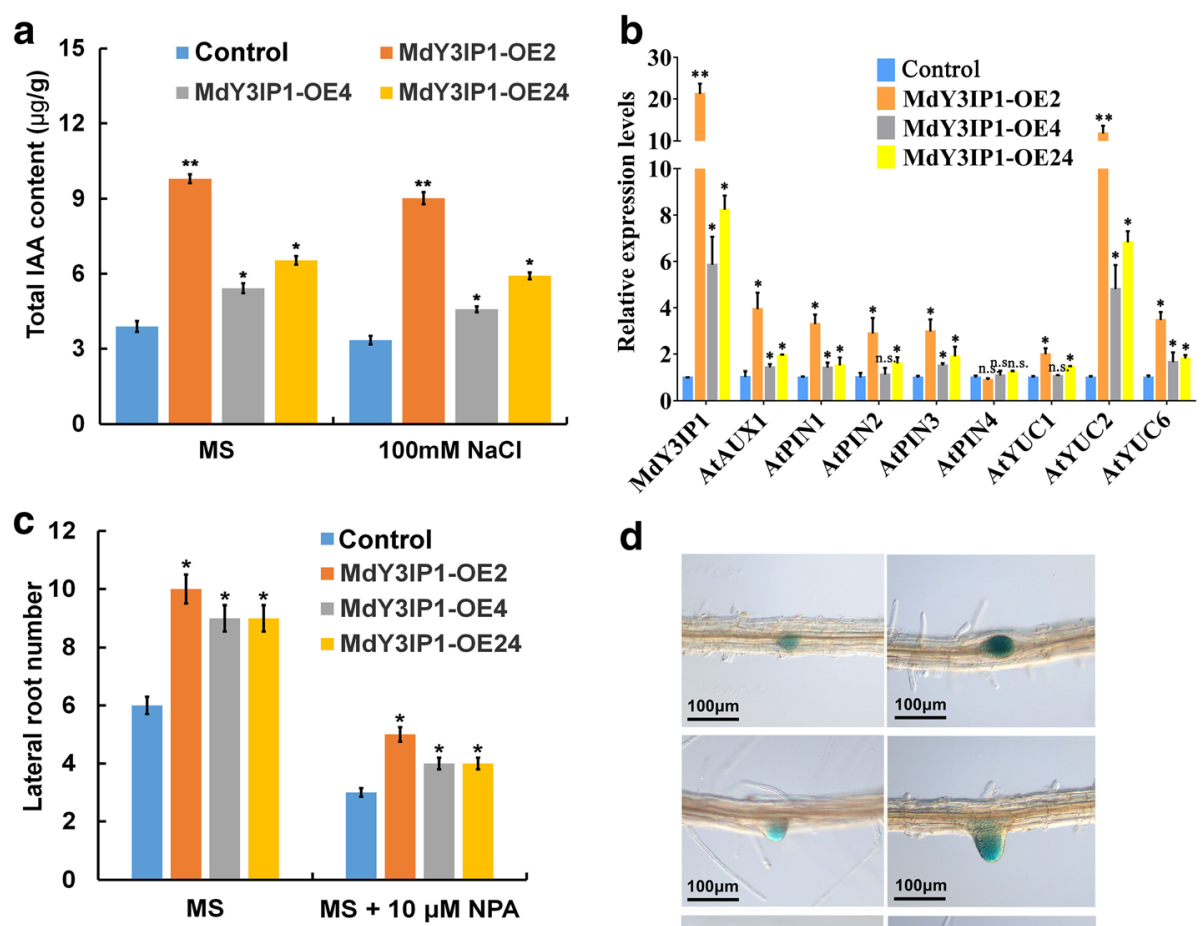

d

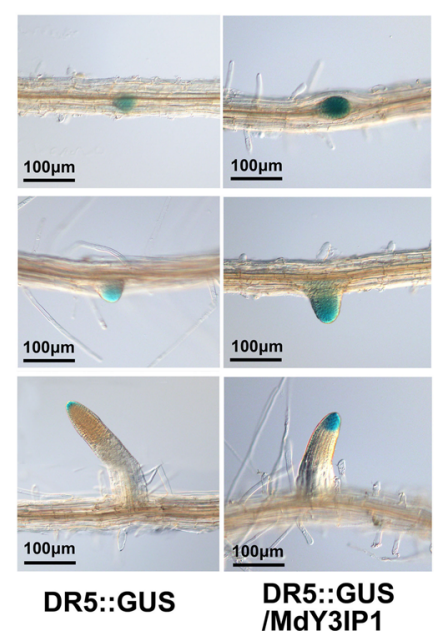

Fig. 5 MdY3IP1 promotes auxin accumulation in lateral roots and lateral root primordia of transgenic Arabidopsis plants. a Total IAA content in the roots of 2-week-old control and MdY3IP1 transgenic Arabidopsis plants. b Transcripts levels of auxin influx carriers (AtAUX1), efflux carriers (AtPIN1, AtPIN2, and AtPIN3), and biosynthetic YUCCA genes (AtYUC1, AtYUC2 and AtYUC6), in control and MdY3IP1 transgenic Arabidopsis by qPCR assay. $\mathbf{c}$ Effect of the auxin transport inhibitor NPA on LR initiation in control and MdY3IP1 transgenic Arabidopsis plants. 5-day-old seedlings were transferred to MS medium supplemented with NPA (10 $\mu \mathrm{M})$. After 10-days growth, the emerged LRs of 10 to 13 seedlings were counted. $\mathbf{d}$ Histochemical staining of GUS activity in LR primordia (top and middle panel) and LR (bottom panel) at three different stages. 10-day-old Arabidopsis plants expressing DR5:GUS in control (left) or MdY3IP1 transgenic plants (right) were used for GUS staining for $8 \mathrm{~h}$. Bars $=100 \mu \mathrm{m}$. e Histochemical staining of GUS activity in primary roots. 10-day-old Arabidopsis plants expressing DR5:GUS in control (left) or MdY3IP1 transgenic plants (right) were used for GUS staining for $8 \mathrm{~h}$. Bars $=100 \mu \mathrm{m}$. Note: In $\mathbf{a}, \mathbf{b}, \mathbf{c}$, data are shown as the mean $\pm \mathrm{SE}$, based on more than nine replicates. Statistical significance was determined using Student's $t$ test. n.S., $P>0.01 ;{ }^{* P}<0.01 ;{ }^{* * P}<0.001$

primary root length were observed between the control and $M d Y 3 I P 1$ transgenic plants (Additional file 4). These results suggested that MdY3IP1 promotes only the LR development, and NPA could not completely eliminate the preferential LR development in transgenic plants.

As our results suggested that MdY3IP1 mediates LR development in an auxin-dependent manner (Fig. 5a, b), we examined endogenous auxin levels with the DR5::GUS reporter; this reporter localizes to regions with high auxin content. GUS staining revealed that the reporter was expressed in root tips of primary roots, as well as in LR and their primordia, independent of genotype (Fig. 5d, e). Interestingly, GUS staining was dramatically increased in emerged LR and LR primordia of MdY3IP1 transgenic Arabidopsis (Fig. 5d). However,
MdY3IP1 did not alter DR5::GUS expression in the primary root tips (Fig. 5e). These results suggest that MdY3IP1 especially affects endogenous auxin levels in LRs.

In summary, these results support that $M d Y 3 I P 1$-expression promotes LR development by influencing auxin local biosynthesis and polar transport.

\section{Exogenous auxin application mimics the effect of MdY3IP1-expression in Arabidopsis roots}

IAA (Indole-3-acetic acid) is a form of auxin that occurs naturally and is commonly used in studies of auxin homeostasis, transport, and response during LR initiation and development [33]. To confirm the role of high auxin levels in the LR phenotype of MdY3IP1-expressing Arabidopsis plants, we included IAA $(0.1 \mu \mathrm{M})$ in our 
growth medium. IAA application mimicked the effect of $M d Y 3 I P 1$ expression in the LR developmental phenotype of the control and MdY3IP1-expressing Arabidopsis plants grown in MS media with or without $\mathrm{NaCl}$ (Fig. 6a, b). No significant difference was observed in primary root development when applying IAA (Fig. 6b). These results further support that MdY3IP1 affects primary and lateral root development under salt stress condition through its effects on auxin homeostasis.

\section{Discussion}

PSI (plastocyanin-ferredoxin oxidoreductase), a participant of the photosynthetic electron transport chain, is one of the largest bioenergetics complexes known to date $[6,7,13,14]$. Although PSI assembly steps are still not fully understood, several auxiliary protein factors have been proven to participate in that process [16-18]. The Y3IP1 protein specifically interacts with Ycf3 to promote PSI assembly in Arabidopsis and tobacco [17]. In this study, we identified the apple homolog of Y3IP1, MdY3IP1, and showed that ectopically expressing it in Arabidopsis lead to early flowering and enhanced salt tolerance phenotypes. These findings provide insights into new functions and potential additional mechanisms of Y3IP1 action in addition to its role in the PSI assembly.

Y3IP1 is an approximately $24 \mathrm{kDa}$ nuclear-encoded protein that is post-translationally imported into chloroplasts. It closely associates with the thylakoid membrane
$[13,17]$, most likely through a putative $\alpha$-helical transmembrane domain on its C-terminal portion (Additional file 5) [13]. In Arabidopsis, Y3IP1 overexpression strongly and specifically increased PSI accumulation [17], suggesting that Y3IP1 is required for PSI assembly. It is known that photosynthesis-derived sugar metabolism promotes flowering by regulating $F T$ gene expression [29, 34]. Thus, it was not surprising that MdY3IP1-expression in Arabidopsis improved photosynthetic carbohydrate synthesis by PSI but not PSII accumulation, consequently altering the expression of flowering-associated genes including $F L C, S O C 1$ and $F T$, and causing an early flowering phenotype (Figs. 1, 2 and 7; Table 1). In addition, the y3ip1 mutant phenotype in Arabidopsis suggested that Y3IP1 promotes plant growth and development [17]. This result was in line with our early flowering phenotype.

Plant growth and development are adversely affected by multiple stresses, which generate various types of dangerous cell-damaging ROS [35-37]. Plants have evolved different types of defense against the adverse effects of ROS, including production of antioxidant compounds and various kinds of defense proteins. For example, chloroplasts contain high concentrations of antioxidant molecules, like ascorbate and glutathione. Additionally, several types of antioxidant enzymes, including ascorbate peroxidase (APX) and superoxide dismutase (SOD), play critical roles in the scavenging of
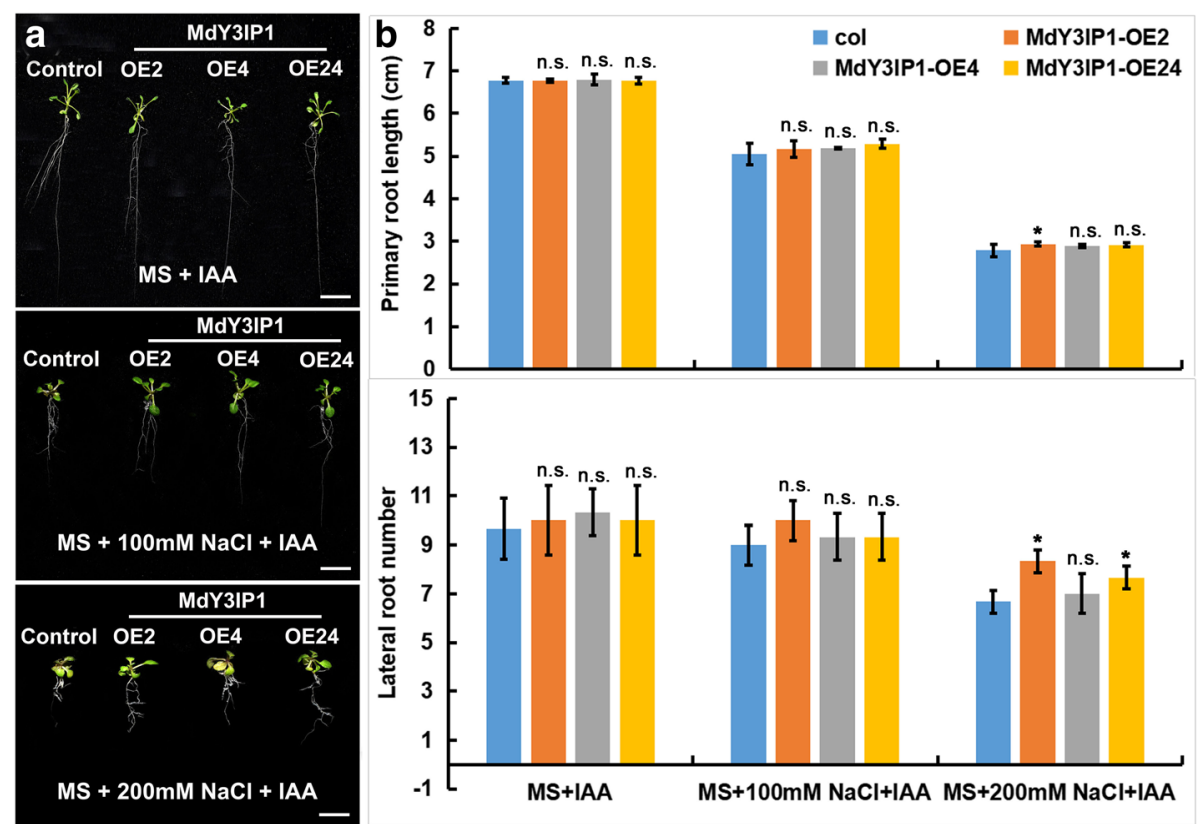

Fig. 6 IAA application mimics the effect of MdY3IP1-expression in Arabidopsis roots. a Root architecture of control and MdY3IP1 transgenic Arabidopsis plants grown on MS medium supplemented with 0, 100 and $200 \mathrm{mM} \mathrm{NaCl}$, respectively. Bars $=1 \mathrm{~cm}$. b primary root length and LR numbers of control and MdY3IP1 transgenic Arabidopsis plants as indicated in (a). Note: In $\mathbf{b}$, data are shown as the mean \pm SE, based on more than nine replicates. Statistical significance was determined using Student's $t$ test. n.S., $P>0.01$; ${ }^{*} P<0.01$ 


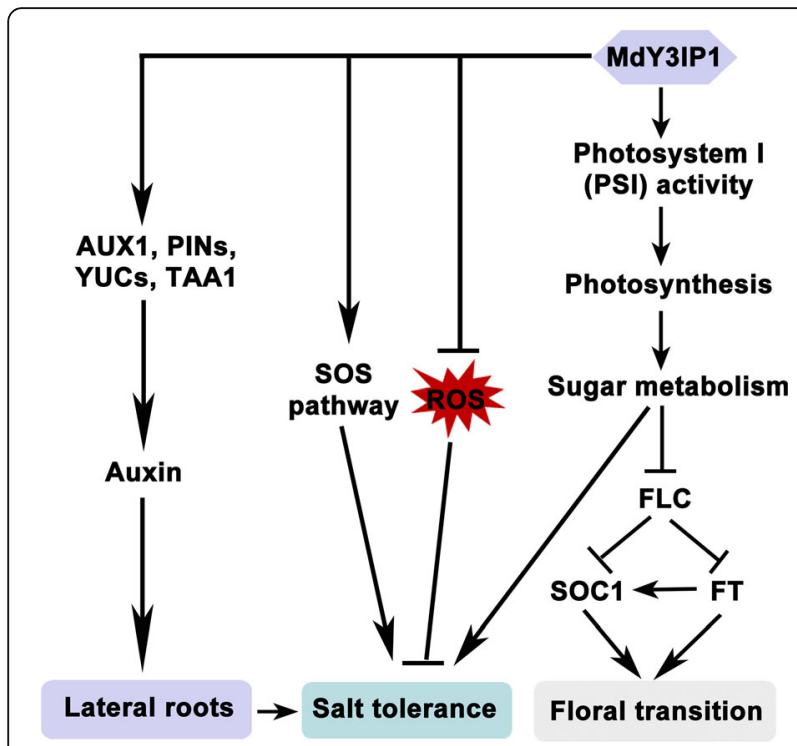

Fig. 7 Working model for MdY3IP1 function in the regulation of photoperiodic flowering and salt tolerance. In the current working model, MdY3IP1 controls floral transition by altering the levels of sugar metabolism, and thereby influencing the expression of floweringassociated genes. MdY3IP1-expression reduces ROS production in plant cells, and promotes LR development by regulating auxin biosynthesis and transport. Additionally, it increases the expression of genes in the SOS pathway, and elevates the levels of sugar metabolism, leading to an increase in tolerance to salt stress in plants

ROS [38, 39]. MdY3IP1 expression was induced in apple plantlets by various abiotic stress treatments, including salinity, low temperature and drought stress (Fig. 3b; Additional file 3). Interestingly, $M d Y 3 I P 1$-expression in Arabidopsis enhanced salt tolerance by reducing ROS production (Fig. 4). Previous work showed that Y3IP1 overexpression in Arabidopsis increased its tolerance to salt and oxidative stresses, leading to the gene named CHLOROPLAST PROTEIN-ENHANCING STRESS TOLERANCE (CEST) [28]. The improvement in salt tolerance was attributed to elevated PSI levels in Y3IP1overexpressing plants [13].

It was previously reported that plant sugars play crucial roles during abiotic stress response [40]. Similarly, we speculate that MdY3IP1-expression increases salt tolerance by improving sugar metabolism (Fig. 7). We also found that MdY3IP1-expression reduced ROS accumulation and enhance salt tolerance (Figs. 4 and 7). Remarkably, salt overly sensitive (SOS) pathway genes, including AtSOS1, AtSOS2, and AtSOS3, were upregulated in MdY3IP1-overexpressing Arabidopsis (Additional file 6), implying that MdY3IP1 increases salt tolerance partly through the SOS pathway (Fig. 7).

The root system architecture (RSA) is plastic and dynamic, allowing plants to respond to various environmental stresses [41, 42]. RSA modifications in salt challenged plants include modulation of LR development by the auxin homeostasis-regulating transcription factor, WRKY46 [33]. Another example is the improved growth observed in Trichoderma spp.-treated Arabidopsis seedlings grown under salt stress, which correlates with enhanced root development, osmolite production, and $\mathrm{Na}^{+}$elimination through root exudates [43]. Interestingly, expression of MdY3IP1 in Arabidopsis promoted LR development by influencing local auxin biosynthesis and polar transport (Fig. 5). LRs initiate from cell divisions in the pericycle of the primary root. The root primordium forms a meristem and pushes its way through the cell layers to generate the LR [44]. The phytohormone auxin is known to be a key regulator of LR development. Altering its content, biosynthesis, distribution, or downstream signaling pathways deeply influences LR formation [45-47]. Disturbance of normal auxin distribution in LR founder cells using polar auxin transport inhibitor NPA is sufficient to completely block LR initiation [48]. We propose that phytohormones exert their effect on LR formation partially through the function of Y3IP1-like genes. Hence, MdY3IP1-expression in Arabidopsis may enhance root development through auxin-regulated pathways, leading to RSA changes and higher tolerance to salt stress. Our proposed work model describes how MdY3IP1-expression confers earlyflowering and salt tolerance phenotypes to plants (Fig. 7).

Here, we showed that overexpression of MdY3IP1 conferred early-flowering and salt tolerance phenotypes. These observations suggest that plant growth and stress resistance can be affected by the regulation of the MdY3IP1 gene. Further molecular and genetic approaches will accelerate our knowledge of MdY3IP1 functions in PSI complex formation and plants stress resistance, and inform strategies for creating transgenic crop varieties with early maturity and high-resistant to adverse environmental conditions.

\section{Conclusion}

In this study, we showed that MdY3IP1 encodes an apple protein highly homologous to the Arabidopsis Y3IP1 (AtY3IP1) PSI assembly factor. Ectopic expression of MdY3IP1 triggered early flowering and enhanced salt tolerance phenotypes in Arabidopsis plants. Further analyses found that MdY3IP1 controlled floral transition by improving photosynthetic derived sugar metabolism in plant cells, thereby influencing the expression of flowering-associated genes. Additionally, MdY3IP1 enhanced salt stress tolerance, by reducing ROS levels, and promoting LR development through auxin biosynthesis and transport pathways. Our observations confirm that plant growth and stress resistance are affected by 
the regulation of Y3IP1-like genes. It accelerates our knowledge of Y3IP1 functions in PSI complex formation, plant stress resistance, and growth/development by more molecular and genetic approaches, and provides strategies for creating genetically modified crops with early maturity and high-resistant to stress.

\section{Methods}

Plant materials, growth conditions, and treatments

Arabidopsis thaliana ecotype 'Columbia' (col) was used in this study. Arabidopsis seeds were surface-sterilized for $5 \mathrm{~min}$ in $75 \%$ alcohol and $20 \mathrm{~min}$ in $10 \%$ sodium hypochlorite, then washed six times with sterile water. Seeds were plated on Murashige and Skoog (MS) solid medium containing $1 \%(w / v)$ sucrose and $0.7 \%(\mathrm{w} / \mathrm{v})$ agar. After vernalizing at $4{ }^{\circ} \mathrm{C}$ for 3 days, seeds germinated at $22{ }^{\circ} \mathrm{C}$ under a 16 -h light/8-h dark photoperiod. Subsequently, 2-week-old seedlings were transferred to soil for further studies.

For the N-1-naphthylphtha-lamic acid (NPA) treatment, 5-day-old seedlings were transferred on MS supplemented with $5 \mu \mathrm{M}$ of the chemical (Greyhound Chem Service, Birkenhead, UK). These plates were placed vertically, and grown at $22{ }^{\circ} \mathrm{C}$ under a 16-h light/ 8-h dark photoperiod for 10 days.

\section{Subcellular localization}

The full-length ORF of MdY3IP1 was cloned and inserted in a $\mathrm{T}$ vector (1258) digested with $\mathrm{XcmI}$ (NEB, Beijing) using the T4 ligase (TaKaRa, Japan). The $p$ CaMV35S::MdY3IP1-GFP recombinant plasmid was transiently transformed into protoplasts extracted from young apple leaves. The protoplast extraction methods were described before [49]. Images were obtained using a confocal laser scanning microscope (LSM510; Carl Zeiss, Oberkochen, Germany). GFP signals were collected using an emission filter of BP505-530 nm, with excitation at $488 \mathrm{~nm}$, and autoflorencence red signals (MitoTracker stain) were obtained using BP $585-615 \mathrm{~nm}$, with excitation at $543 \mathrm{~nm}$.

\section{Quantitative RT-PCR (qPCR) assays}

Total RNA extraction from the Arabidopsis plants indicated in each $\mathrm{qPCR}$ assay was performed using the RNeasy plant mini-prep kit (TIANGEN, Beijing) according to the manufacturer's instructions. After digestion with DNase I (TaKaRa, Dalian), $1 \mu \mathrm{g}$ purified RNA was converted into single stranded cDNA using the M-MLV reverse transcriptase (TaKaRa, Dalian). qPCR reactions were previously described [49]. The housekeeping gene AteIF4a was used as control. More than 9 biological replicates were performed for each experiment. The primers used for qRT-PCR are listed in the Additional file 7.

Heterologous transformation of MdY3IP1 into Arabidopsis For Arabidopsis transformation, the $35 S-m y c$ and 35S::MdY3IP1-myc recombinant plasmids were introduced into ecotype Columbia (Col-0) using the floral dip method [50]. The Agrobacterium strain used was GV3101. After two generation of selection in hygromycin $(30 \mathrm{mg} / \mathrm{L})$, seeds of screened transgenic plants were harvested. Homozygotes were identified with a qPCR assay. Homozygous transgenic seeds from individual plants were used in further experiments.

\section{Isolation of thylakoids and immunoblotting assays}

Thylakoid proteins of control and MdY3IP1 transgenic Arabidopsis were isolated from leaves using published procedures [51]. For immunoblotting assays, samples were normalized to chlorophyll, electrophoretically separated on an SDS-polyacrylamide gels [52], and transferred to Hybond-P polyvinylidene difluoride membranes (GE Healthcare) using standard protocols. Immunoblot detection was performed with specific antibodies using the ECL PLUS system (Signa $\mathrm{VH} / \mathrm{i}$; GE Healthcare, Waukesha, WI, USA). Polyclonal antibodies against ACTIN, myc, PsaA, PsaD, PsaF, and D1 were prepared from rabbit by the Abmart Company (Shanghai, China). Immunoblotting assays were described before [53].

\section{Measurement of chlorophyll content and chlorophyll fluorescence difference absorption spectroscopy}

Fresh Arabidopsis leaves (1.0 g) were homogenized with a plant tissue homogenizer in $20 \mathrm{ml}$ of $95 \%$ ethanol $\left(v / \mathrm{v}\right.$ in $\left.\mathrm{ddH}_{2} \mathrm{O}\right)$. The homogenized samples were then centrifuged at $12,000 \mathrm{~g}$ for $10 \mathrm{~min}$ at $4{ }^{\circ} \mathrm{C}$. The supernatants were diluted 10-fold using 95\% ethanol. Chlorophyll content was measured by a UV/ visible spectrophotometer (OPTIZEN POP, Mecasys). An SPDA-502 chlorophyll meter (KONICA MINOLTA) was used for direct measurements.

Chlorophyll fluorescence was recorded with a pulse amplitude modulated fluorimeter (Dual-PAM-100; Heinz Walz) on intact Arabidospis plants, at room temperature. Plants were dark adapted for $1 \mathrm{~h}$ prior to determination of the maximum PSII quantum efficiency $(\mathrm{Fv} / \mathrm{fm})$ and non-photochemical quenching (NPQ). The contents of PSI were determined in thylakoid preparations as previously described [50]. PSI was quantified by determining the P700 difference absorption signal sat 830 to $870 \mathrm{nmin}$ in solubilized thylakoids, using the Dual-PAM instrument [54]. 
Measurement procedures and deconvolution methods were previously described $[55,56]$.

Starch staining and determination of total soluble sugars 4-week-old leaves of control and MdY3IP1 transgenic Arabidopsis were immersed in a solution of Lugol containing $0.2 \%$ iodine and $2 \%$ potassium iodine, and incubated for about $1 \mathrm{~min}$. They were then washed with $\mathrm{ddH}_{2} \mathrm{O}$ and placed in a mounting solution $(7.5 \mathrm{~g}$ gum arabic, $100 \mathrm{~g}$ chloroacetaldehyde, $5 \mathrm{~mL}$ glycerol, and $60 \mathrm{~mL}$ water). The Lugol-stained leaves were photographed with a standard camera.

For determining total soluble sugars content, fresh Arabidopsis leaves $(0.2 \mathrm{~g})$ were boiled in water for $30 \mathrm{~min}$ for extraction, and the sugar levels were determined using the anthrone reagent with glucose as a standard. The absorbance was read at $630 \mathrm{~nm}$, and the sugar concentration was determined using a glucose standard curve.

\section{Measurement of ROS}

2-week-old seedlings of control and MdY3IP1 transgenic Arabidopsis were treated in liquid MS with or without $200 \mathrm{mM} \mathrm{NaCl}$ for $5 \mathrm{~h}$ before the seedlings were used for follow-up staining analysis. 3', 3'-diaminobenzidine (DAB) and nitroblue tetrazolium (NBT) staining were conducted as described before [37]. For $2^{\prime}, 7^{\prime}$-dichlorodihydrofluorescin diacetate (DCFH-DA) staining to detect $\mathrm{H}_{2} \mathrm{O}_{2}$, protoplasts isolated from Arabidopsis leaves were incubated in a buffer containing $50 \mathrm{mM}$ DCFH-DA (Sigma-Aldrich) and $20 \mathrm{mM}$ K-phosphate at $\mathrm{pH} 6.0$ in darkness for $10 \mathrm{~min}$. These protoplasts were then photographed using a confocal laser scanning microscope (LSM510; Carl Zeiss, Oberkochen, Germany) with an excitation at $488 \mathrm{~nm}$. The intensity of the fluorescent signals was quantified using the ImageJ software.

\section{Histochemical staining of GUS activity}

GUS activity staining was conducted as described previously [57]. After incubating the plant material at $37{ }^{\circ} \mathrm{C}$ for $8 \mathrm{~h}$ in the dark, individual representative seedlings were photographed with a confocal laser scanning microscope (Zeiss LSM 510 META, Jena, Germany). A total of 20 to 30 Arabidopsis plants per genotype were imaged in this experiment.

\section{Auxin content measurement}

2-week-old seedlings of the control and MdY3IP1 transgenic Arabidopsis were harvested and ground to powder in liquid nitrogen. For each sample, approximate $0.1 \mathrm{~g}$ powder were resuspended in pre-cooling $80 \%$ methanol and mixed immediately. The samples were kept at $4{ }^{\circ} \mathrm{C}$ protected from light before $0.8 \mathrm{ng}$ $\left[{ }^{13} \mathrm{C}\right]$-IAA was added. IAA content measurement was performed using GC-QQQ (Agilent, 7000A).

\section{Statistical analysis}

Samples were analyzed in triplicates, and the data expressed as the mean \pm standard error (SE) unless noted otherwise. Statistical significance was determined using Student's $t$-test. A difference at $P \leq 0.01$ was considered significant (*), and $P \leq 0.001$ was considered extremely significant ${ }^{(* *)}$.

\section{Additional files}

Additional file 1: Figure S1. Analysis of the deduced amino acid of MdY3IP1. (DOC $1372 \mathrm{~kb}$ )

Additional file 2: Figure S2. Early flowering phenotype in the MdY3IP1expressing transgenic Arabidopsis plants under short-days condition. (DOC $702 \mathrm{~kb}$ )

Additional file 3: Figure S3. Expression of $M d Y 3 / P 1$ under $4{ }^{\circ} \mathrm{C}, 2 \%$ PEG and $100 \mu \mathrm{M}$ ABA. (ZIP $292 \mathrm{~kb}$ )

Additional file 4: Figure S4. Effect of the auxin transport inhibitor NPA on primary root length in control and MdY3IP1 transgenic Arabidopsis plants. (ZIP 476 kb)

Additional file 5: Figure S5. The deduced functional domains of MdY3IP1 protein. (DOC $44 \mathrm{~kb}$ )

Additional file 6: Figure S6. Expression levels of AtSOS1, AtSOS2 and AtSOS3 in the control and MdY3IP1 transgenic Arabidopsis plants by qPCR assay. (ZIP $275 \mathrm{~kb})$

Additional file 7: Table S1. The primers used for RT-PCR and qRT-PCR in this study. (PDF $18 \mathrm{~kb}$ )

\section{Abbreviations}

CEST: Chloroplast protein-enhancing stress tolerance; LHCl: Light-harversting complex l; NPA: N-1-naphthylphtha-lamic acid; NPQ: Non-photochemical quenching; PGR5: PROTON GRADIENT REGULATION 5; PSI: Photosystem I; qRT-PCR: Quantitative reverse-transcription PCR; ROS: Reactive oxygen species; Y3IP1: Ycf3-interacting protein; Ycf3: Hypothetical chloroplast reading frame number 3

\section{Acknowledgements}

We thank Prof. Hui-Yuan Gao (National Key Laboratory of Crop Biology; College of Life Sciences, Shandong Agricultural University, Tai-An, Shandong, China) for providing protein antibodies including PsaA and D1.

\section{Funding}

This work was supported by the National Natural Science Foundation of China (31601728, 31325024 and 31430074), Ministry of Education of China (IRT15R42), the Natural Science Foundation of Shandong Province (ZR2016CQ13 and SDAIT-06-03), Young Scientists Funds of Shandong Agricultural University (564024), and Youth Science and Technology Innovation Fund of Shandong Agricultural University (24024).

Availability of data and materials

The datasets supporting the conclusions of this article are included within the article and its additional files. The MdY3IP1 sequence is available at apple genome database (https://iris.angers.inra.fr/gddh13/the-apple-genomedownloads.html).

\section{Authors' contributions}

YJH and DGH conceived and designed the experiments. DGH, JQY, CHS QYZ and JHW performed the experiments. DGH and JQY wrote the article. All authors read and approved the final manuscript. 


\section{Ethics approval and consent to participate}

Not applicable

\section{Consent for publication \\ Not applicable}

\section{Competing interests}

The authors declare that they have no competing interests.

\section{Publisher's Note}

Springer Nature remains neutral with regard to jurisdictional claims in published maps and institutional affiliations.

\section{Author details}

'National Key Laboratory of Crop Biology, College of Horticulture Science and Engineering, Shandong Agricultural University, Tai-An, Shandong 271018, China. ${ }^{2}$ National Key Laboratory of Crop Biology, MOA Key Laboratory of Horticultural Crop Biology and Germplasm Innovation, College of Horticulture Science and Engineering, Shandong Agricultural University, Tai-An, Shandong 271018, China.

\section{Received: 14 September 2017 Accepted: 11 January 2018}

\section{Published online: 20 January 2018}

\section{References}

1. Peltier JB, Cai Y, Sun Q, Zabrouskov V, Giacomelli L, Rudella A, Ytterberg AJ Rutschow H, van Wijk KJ. The oligomeric stromal proteome of Arabidopsis thaliana chloroplasts. Mol Cell Proteomics. 2006;5:114-33.

2. Biswal B, Krupinska K, Biswal UC: Plastid development in leaves during growth and senescence. 2013.

3. Buchanan BB, Gruissem W, Jones RL. Biochemistry and molecular biology of plants. New York: Wiley; 2015. p. 1367.

4. Jordan P, Fromme P, Witt HT, Klukas O, Saenger W, Krauß N. Threedimensional structure of cyanobacterial photosystem I at $2.5 \AA$ resolution. Nature. 2001:411:909-17.

5. Nelson N, Ben-Shem A. The complex architecture of oxygenic photosynthesis. Nat Rev Mol Cell Biol. 2004;5:1-12.

6. Amunts A, Drory O, Nelson N. The structure of a plant photosystem I supercomplex at 3.4 $\AA$ resolution. Nature. 2007:447:58-63.

7. Amunts A, Nelson N. Plant photosystem I design in the light of evolution. Structure. 2009:17:637-50.

8. Asada K. The water-water cycle as alternative photon and electron sinks. Philos T R Soc B Biol Sci. 2000:355:1419-31.

9. Munekage Y, Hojo M, Meurer J, Endo T, Tasaka M, Shikanai T. PGR5 is involved in cyclic electron flow around photosystem I and is essential for photoprotection in Arabidopsis. Cell. 2002;110:361-71.

10. Tikkanen M, Mekala NR, Aro EM. Photosystem II photoinhibition-repair cycle protects Photosystem I from irreversible damage. BBA-Bioenergetics. 2014; 1837:210-5.

11. Grotjohann I, Fromme P. Structure of cyanobacterial photo-system I. Photosynth Res. 2005;85:51-72.

12. Ozawa S, Onishi T, Takahashi Y. Identification and characterization of an assembly intermediate subcomplex of Photosystem I in the green alga Chlamydomonas Reinhardtii. J Biol Chem. 2010;285:20072-9.

13. Schöttler MA, Albus CA, Bock R. Photosystem I: its biogenesis and function in higher plants. J Plant Physiol. 2011;168:1452-61.

14. Mazor Y, Borovikova A, Nelson N. The structure of plant photosystem super-complex at $2.8 \AA$ resolution. elife. 2015:4:e07433.

15. Nelson $N$, Junge W. Structure and energy transfer in photosystems of oxygenic photosynthesis. Annu Rev Biochem. 2015;84:659-83.

16. Yang H, Liu J, Wen X, Lu C. Molecular mechanism of photosystem I assembly in oxygenic organisms. BBA-Bioenergetics. 2015;1847:838-48.

17. Albus CA, Ruf S, Schottler MA, Lein W, Kehr J, Bock R. Y3IP1, a nucleusencoded thylakoid protein, cooperates with the plastid-encoded YCF3 protein in photosystem I assembly of tobacco and Arabidopsis. Plant Cell. 2010;22:2838-55

18. Krech K, Ruf S, Masduki FF, Thiele W, Bednarczyk D, Albus CA, Tiller N, Hasse C, Schöttler MA, Bock R. The plastid genome-encoded Ycf4 protein functions as a nonessential assembly factor for Photosystem I in higher plants. Plant Physiol. 2012;159:579-91.
19. Wilde A, Härtel H, Hübschmann T, Hoffmann P, Shestakov SV, Börner T. Inactivation of a Synechocystis sp strain PCC 6803 gene with homology to conserved chloroplast open reading frame 184 increases the Photosystem II-to-Photosystem I ratio. Plant Cell. 1995;7:649-58.

20. Boudreau E, Takahashi Y, Lemieux C, Turmel M, Rochaix JD. The chloroplast ycf3 and ycf4 open reading frames of Chlamydomonas Reinhardtii are required for the accumulation of the Photosystem I complex. EMBO J. 1997; 16:6095-104.

21. Naver H, Boudreau E, Rochaix JD. Functional studies of Ycf3: its role in assembly of Photosystem I and interactions with some of its subunits. Plant Cell. 2001:13:2731-45.

22. Stöckel J, Bennewitz S, Hein P, Oelmüller R. The evolutionarily conserved tetratrico peptide repeat protein pale yellow green 7 is required for Photosystem I accumulation in Arabidopsis and copurifies with the complex. Plant Physiol. 2006:141:870-8.

23. Fristedt $\mathrm{R}$, Williams-Carrier R, Merchant SS, Barkan A. A thylakoid membrane protein harboring a DnaJ-type zinc finger domain is required for photosystem I accumulation in plants. J Biol Chem. 2014;289:30657-67.

24. Heinnickel M, Kim RG, Wittkopp TM, Yang W, Walters KA, Herbert SK, Grossman AR. Tetratricopeptide repeat protein protects photosystem I from oxidative disruption during assembly. Proc Natl Acad Sci. 2016:113:2774-9.

25. Wang YW, Chen SM, Wang WJ, Huang XQ, Zhou CF, Zhuang Z, Lu S. The dnaJ-like zinc finger domain protein PSA2 affects light acclimation and chloroplast development in Arabidopsis thaliana. Front Plant Sci. 2016;7:360.

26. Järvi S, Suorsa M, Tadini L, Ivanauskaite A, Rantala S, Allahverdiyeva Y, Aro EM. FtsH facilitates proper biosynthesis of photosystem I in Arabidopsis thaliana. Plant Physiol. 2018;176(1):1-11.

27. Shen J, Williams-Carrier R, Barkan A. PSA3, a protein on the stromal face of the thylakoid membrane, promotes photosystem I accumulation in cooperation with the assembly factor PYG7. Plant Physiol. 2017; https://doi. org/10.1104/pp17.00524.

28. Yokotani N, Higuchi M, Kondou Y, Ichikawa T, Iwabuchi M, Hirochika H, Matsui M, Oda K. A novel chloroplast protein CEST induces tolerance to multiple environmental stresses and reduces photooxidative damage in transgenic Arabidopsis. J Exp Bot. 2010;62:557-69.

29. Seo PJ, Ryu J, Kang SK, Park CM. Modulation of sugar metabolism by an INDETERMINATE DOMAIN transcription factor contributes to photoperiodic flowering in Arabidopsis. Plant J. 2011;65:418-29.

30. Bolouri Moghaddam MR, Van den Ende W. Sugars, the clock and transition to flowering. Front Plant Sci. 2013;4:22.

31. Baxter A, Mittler R, Suzuki N. ROS as key players in plant stress signalling. J Exp Bot. 2014;65:1229-40.

32. Oukarroum A, Bussotti F, Goltsev V, Kalaji HM. Correlation between reactive oxygen species production and photochemistry of photosystems I and II in Lemna Gibba L. plants under salt stress. Environ Exp Bot. 2015;109:80-8.

33. Ding ZJ, Yan JY, Li CX, Li GX, Wu YR, Zheng SJ. Transcription factor WRKY46 modulates the development of Arabidopsis lateral roots in osmotic/salt stress conditions via regulation of ABA signaling and auxin homeostasis. Plant J. 2015:84:56-69.

34. Ortiz-Marchena Ml, Albi T, Lucas-Reina E, Said FE, Romero-Campero FJ, Cano $B$, Valverde F. Photoperiodic control of carbon distribution during the floral transition in Arabidopsis. Plant Cell. 2014;26:565-84.

35. Vinocur B, Altman A. Recent advances in engineering plant tolerance to abiotic stress: achievements and limitations. Curr Opin Plant Biotech. 2005;16:123-32.

36. Takahashi S, Murata N. How do environmental stresses accelerate photoinhibition? Trends Plant Sci. 2008;13:178-82.

37. Hu DG, Ma QJ, Sun CH, Sun MH, You CX, Hao YJ. Overexpression of MdSOS2L1, a CIPK protein kinase, increases the antioxidant metabolites to enhance salt tolerance in apple and tomato. Physiol Plantarum. 2016;156:201-14.

38. Miller G, Suzuki N, Rizhsky L, Hegie A, Koussevitzky S, Mittler R. Double mutants deficient in cytosolic and thylakoid ascorbate peroxidase reveal a complex mode of interaction between reactive oxygen species, plant development, and response to abiotic stresses. Plant Physiol. 2007;144 $1777-85$.

39. Azarabadi S, Abdollahi H, Torabi M, Salehi Z, Nasiri J. ROS generation, oxidative burst and dynamic expression profiles of ROS-scavenging enzymes of superoxide dismutase (SOD), catalase (CAT) and ascorbate peroxidase (APX) in response to Erwinia amylovora in pear (Pyrus Communis L). Eur J Plant Pathol. 2017;147:279-94.

40. Keunen ELS, Peshev D, Vangronsveld J, Van Den Ende WIM, Cuypers ANN. Plant sugars are crucial players in the oxidative challenge during 
abiotic stress: extending the traditional concept. Plant Cell Environ. 2013;36:1242-55.

41. Hu H, Xiong L. Genetic engineering and breeding of drought-resistant crops. Annu Rev Plant Biol. 2014;65:715-41.

42. Fang Y, Xiong L. General mechanisms of drought response and their application in drought resistance improvement in plants. Cell Mol Life Sci. 2015;72:673-89.

43. Contreras-Cornejo HA, Macías-Rodríguez L, Alfaro-Cuevas R, López-Bucio J. Trichoderma spp. improve growth of Arabidopsis seedlings under salt stress through enhanced root development, osmolite production, and $\mathrm{Na}+$ elimination through root exudates. Mol Plant Microbe In. 2014;27:503-14.

44. Malamy JE, Benfey PN. Organisation and cell differentiation in lateral roots of Arabidopsis Thaliana. Development. 1997;124:33-44.

45. Brady SM, Sarkar SF, Bonetta D, MCCourt P. The ABSCISICACID INSENSITIVE 3 (ABI3) gene is modulated by farnesylation and is involved in auxin signaling and lateral root development in Arabidopsis. Plant J. 2003;34:67-75.

46. Tian C, Muto H, Higuchi K, Matamura T, Tatematsu K, Koshiba T, Yamamoto $K T$. Disruption and overexpression of auxin response factor 8 gene of Arabidopsis affect hypocotyl elongation and root growth habit, indicating its possible involvement in auxin homeostasis in light condition. Plant $\mathrm{J}$. 2004;40:333-43.

47. Tsuchisaka A, Theologis A. Unique and overlapping expression patterns among the Arabidopsis 1-amino-cyclopropane-1-carboxylate synthase gene family members. Plant Physiol. 2004;136:2982-3000.

48. Casimiro I, Marchant A, Bhalerao RP, Beeckman T, Dhooge S, Swarup R, Bennett M. Auxin transport promotes Arabidopsis lateral root initiation. Plant Cell. 2001;13:843-52.

49. Hu DG, Sun CH, Ma QJ, You CX, Cheng L, Hao YJ. MdMYB1 regulates anthocyanin and malate accumulation by directly facilitating their transport into vacuoles in apples. Plant Physiol. 2016;170:1315-30.

50. Clough SJ, Bent AF. Floral dip: a simplified method for Agrobacteriummediated transformation of Arabidopsis thaliana. Plant J. 1998;16:735-43.

51. Schöttler MA, Kirchhoff H, Weis E. The role of plastocyanin in the adjustment of the photosynthetic electron transport to the carbon metabolism in tobacco. Plant Physiol. 2004;136:4265-74.

52. Laemmli UK. Cleavage of structural proteins during the assembly of the head of bacteriophage T4. Nature. 1970;227:680-5.

53. Hu DG, Sun CH, Zhang QY, An JP, You CX, Hao YJ. Glucose sensor MdHXK1 phosphorylates and stabilizes MdbHLH3 to promote Anthocyanin biosynthesis in apple. PLoS Genet. 2016;12:e1006273.

54. Schöttler MA, Flügel C, Thiele W, Bock R. Knock-out of the plastid-encoded PetL subunit results in reduced stability and accelerated leaf age-dependent loss of the cytochrome b6f complex. J Biol Chem. 2007;282:976-85.

55. Kirchhoff H, Mukherjee U, Galla HJ. Molecular architecture of the thylakoid membrane: lipid diffusion space for plastoquinone. Biochemistry. 2002;41:4872-82.

56. Schöttler MA, Flügel C, Thiele W, Stegemann S, Bock R. The plastomeencoded Psal subunit is required for efficient photosystem I excitation, but not for plastocyanin oxidation in tobacco. Biochem J. 2007;403:251-60

57. Xi J, Xu P, Xiang CB. Loss of AtPDR11, a plasma membrane-localized ABC transporter, confers paraquat tolerance in Arabidopsis thaliana. Plant J. 2012; 69:782-91.

\section{Submit your next manuscript to BioMed Central and we will help you at every step:}

- We accept pre-submission inquiries

- Our selector tool helps you to find the most relevant journal

- We provide round the clock customer support

- Convenient online submission

- Thorough peer review

- Inclusion in PubMed and all major indexing services

- Maximum visibility for your research

Submit your manuscript at www.biomedcentral.com/submit
Biomed Central 